\title{
Diagnostic Value of Serum Biomarkers in Combined Hepatocelluar-Cholangiocarcinoma
}

\author{
Jiu Chen, Youdi Li and Guoyou Yu \\ The First Affiliated Hospital, College of Medicine, Zhejiang University, Hangzhou, China
}

\begin{abstract}
Objective: To explore the value of des-gamma-carboxyprothrombin (DCP), alpha-fetoprotein (AFP) and carbohydrate antigen-199 (CA-199) for diagnosis of combined hepatocellular-cholangiocarcinoma (cHCC-CC).

Study Design: Observational correlational study design.

Place and Duration of Study: The First Affiliated Hospital, College of Medicine, Zhejiang University, Hangzhou, China, from June 2016 to June 2019.

Methodology: Patients with cHCC-CC $(n=26)$ diagnosed by histological examination and immunohistochemical staining were matched as 1:2 to controls $(n=52)$ based on age ( \pm 2 years), gender (male or female), liver texture (cirrhosis or not) and ethnicity. Serum DCP, AFP and CA-199 levels were determined and Youden indices were calculated. Receiver operating characteristic (ROC) curves were generated and areas under the receiver operating characteristic curve (AUC) were calculated.

Results: The levels of DCP, AFP and CA-199 were clearly elevated in the CHCC-CC group relative to controls with p-values of $<0.001,<0.001$ and 0.001 , respectively. Youden index values for DCP, AFP, CA-199, and their combination (DCP+AFP+CA-199) were 0.5, 0.423, 0.173 and 0.673, respectively. The AUC curve values for DCP, AFP, CA-199 and their combination (DCP+AFP+CA-199) were 0.759 (95\% Cl: $0.624-0.894), 0.826$ (95\% Cl: $0.720-0.933), 0.727$ (95\% Cl: $0.613-0.841)$ and 0.862 (95\% Cl: $0.755-0.968)$, respectively.

Conclusion: DCP, AFP and CA-199 have diagnostic value for CHCC-CC, and the combination of DCP, AFP and CA-199 is useful for assisting diagnosis before histological examination. Clinicians should be alert for cHCC-CC when DCP, AFP, or CA-199 levels are abnormal.
\end{abstract}

Key Words: Diagnosis, Des-gamma-carboxyprothrombin, Alpha-fetoprotein, Carbohydrate antigen-199, Combined hepatocelluarcholangiocarcinoma.

How to cite this article: Chen J, Li Y, Yu G. Diagnostic value of serum biomarkers in combined hepatocelluar-cholangiocarcinoma. $J$ Coll Physicians Surg Pak 2020; 30(3):263-267.

\section{INTRODUCTION}

Combined hepatocellular-cholangiocarcinoma (cHCC$\mathrm{CC}$ ) is a rare type of primary liver cancer $(\mathrm{PLC})^{1}$ that originates from hepatic progenitor cells with histological features of hepatocellular carcinoma (HCC) and cholangiocarcinoma (CC). ${ }^{2}$ With the incidence of only $1.3 \%$ of all PLC cases, 1 cHCC-CC is more aggressive and is associated with poorer prognosis, than either $\mathrm{HCC}$ or CC. ${ }^{3}$

Early diagnosis is extremely important for cHCC-CC. Despite its aggressive behaviour and poor prognosis, most cHCC-CC cases are asymptomatic in the early stages and not suitable for surgery when detected, 4

Correspondence to: Dr. Guoyou Yu, Department of Traditional Chinese Medicine, The First Affiliated Hospital, College of Medicine, Zhejiang University, Hangzhou, China

E-mail: chenjiu1982@163.com

Received: August 19, 2019; Revised: December 01, 2019; Accepted: January 30, 2020 while surgical resection is considered the only curative therapeutic option for $\mathrm{CHCC}-\mathrm{CC} .{ }^{1}$ Once a diagnosis of cHCC-CC is established, an immediate aggressive treat-ment strategy is required. 5 Considering the high rate of lymphatic metastasis 6 and the poor prognosis asso-ciated with transplantation, ${ }^{4}$ lymph node dissection is necessary and liver transplantation may not be considered for CHCC-CC.

However, early diagnosis of $\mathrm{CHCC}-\mathrm{CC}$ is elusive and difficult before surgery. ${ }^{2}$ Although diagnostic techniques have been improved substantially in recent decades, diagnosis of CHCC-CC requires confirmation by postoperative pathological examination and immuno-histochemical staining in most patients. ${ }^{7}$ With characteristics of both HCC and CC, majority of CHCC-CC cases are misdiagnosed as either of these entities, regardless of whether diagnosis is by imaging, ${ }^{8}$ biopsy (e.g., sampling only the $\mathrm{HCC}$ or $\mathrm{CC}$ area) or frozen section examination.

Serum biomarkers are useful tools that can assist in diagnosis of numerous cancers. Des-gamma-carboxy- 
prothrombin (DCP) and alpha-fetoprotein (AFP) are the most widely used biomarkers for $\mathrm{HCCs}^{9}$ while carbohydrate antigen-199 (CA-199) has been the most widely used diagnostic biomarker in previous studies of CC.10 However, the diagnostic efficacy of combination of DCP, AFP and CA-199 has been seldom studied before for cHCC-CC.

Considering the importance of achieving diagnosis before surgery and the lack of identification of reliable serum biomarkers for $\mathrm{CHCC}-\mathrm{CC}$ diagnosis to date, the diagnostic efficacy of DCP, AFP and CA-199 were evaluated. This study was aimed to identify reliable serum biomarkers for $\mathrm{CHCC}-\mathrm{CC}$ to improve diagnostic efficacy prior to surgery.

\section{METHODOLOGY}

This is a retrospective observational correlational study. The study was approved by the Ethics Committees of the Hospital. All participants were above 18 years of age. All cHCC-CC and control cases performed at the First Affiliated Hospital, College of Medicine, Zhejiang University from June 2016 to June 2019 were aggregated in a retrospective database from the electronic medical record system.

Variables collected included ethnicity, gender, age, liver texture (cirrhosis or not), the levels of serum markers (DCP, AFP and CA-199) and other quantitative findings. Patients with cHCC-CC diagnosed by histological examination and immunohistochemical staining after surgery $(n=26)$. Patients with $\mathrm{CHCC}-\mathrm{CC}$ were matched $1: 2$ with controls $(n=52)$, based on age ( \pm 2 years), gender (male or female), liver texture (cirrhosis or not) and ethnicity (xanthoderm). Exclusion for the participants was made if the participants had undergone liver transplantation; taking vitamin $\mathrm{K}$ or warfarin within 2 weeks of blood sample collection; exhibited evidence of malignancies other than CHCC-CC; nodules in the liver could not be confirmed (for example, the participants refused surgery or biopsy); or they had received antitumor treatment before serum biomarker measurement.

Serum levels of AFP and CA-199 were analysed using an Abbott i2000SR automatic analyser and associated kits (Abbott Diagnostics, Chicago, Illinois, U.S.A.). Serum levels of DCP were measured by chemiluminescent enzyme immunoassay (Lumipulse $®$ G PIVKA-II; FUJIREBIO INC., Japan). The normal ranges of DCP, AFP and CA199 were $0-40 \mathrm{mAU} / \mathrm{ml}, 0-20 \mathrm{ng} / \mathrm{ml}$ and $0-37 \mathrm{U} / \mathrm{ml}$ respectively.

All statistical analyses were conducted using SPSS 22.0 (IBM, Chicago, US). $p<0.05$ was considered significant. Categorical data are expressed as percentage values. The Mann-Whitney U-test was used to evaluate intergroup differences when quantitative data that were not normally distributed. Receiver operating characteristic (ROC) curve analysis was used to assess the diagnostic efficacy of cHCC-CC. Areas under the receiver operating characteristic curve (AUC) were calculated based on the ROC curves.

\section{RESULTS}

The characteristics of participants with $\mathrm{CHCC}-\mathrm{CC}$ are listed in Table I. Serum biomarkers levels were measured and compared in the control and CHCC-CC groups. The mean levels of DCP, AFP, and CA-199 were $2213.85 \mathrm{mAU} / \mathrm{ml}, 294.26 \mathrm{ng} / \mathrm{ml}$ and $35.33 \mathrm{U} / \mathrm{ml}$ in the cHCC-CC group.

The levels of DCP, AFP, and CA-199 were clearly elevated in the CHCC-CC group relative to controls with the $p$-values of $<0.001,<0.001$ and 0.001 respectively.

To determine the diagnostic efficacy of different serum biomarkers for $\mathrm{CHCC}-\mathrm{CC}$ patients, sensitivity, specificity, Youden index values were calculated and ROC curves were plotted. Both DCP and AFP exhibited good diagnostic efficacy with a Youden index of 0.5 and 0.423

Table I: Characteristics of the studied $\mathrm{cHCC}-\mathrm{CC}$ patients.

\begin{tabular}{|c|c|}
\hline Characteristics & Number of patients \\
\hline \multicolumn{2}{|l|}{ Age } \\
\hline$>50$ years & $80.77 \%(21)$ \\
\hline$<50$ years & $19.23 \%(5)$ \\
\hline \multicolumn{2}{|l|}{ Gender } \\
\hline Male & $88.46 \%(23)$ \\
\hline Female & $11.54 \%(3)$ \\
\hline \multicolumn{2}{|l|}{ Symptomatic } \\
\hline Yes & $30.77 \%(8)$ \\
\hline No & $69.23 \%(18)$ \\
\hline \multicolumn{2}{|l|}{ HBV infection } \\
\hline Yes & $65.38 \%(17)$ \\
\hline No & $34.62 \%(9)$ \\
\hline \multicolumn{2}{|l|}{ Cirrhosis } \\
\hline Yes & $80.77 \%(21)$ \\
\hline No & $19.23 \%(5)$ \\
\hline \multicolumn{2}{|l|}{ Tumor Number } \\
\hline Single & $65.38 \%(17)$ \\
\hline Multiple & $34.62 \%(9)$ \\
\hline \multicolumn{2}{|l|}{ CK19* } \\
\hline Negative & $7.69 \%(2)$ \\
\hline Positive & $92.31 \%(24)$ \\
\hline \multicolumn{2}{|l|}{ GPC-3* } \\
\hline Negative & $26.92 \%(7)$ \\
\hline Positive & $73.08 \%(19)$ \\
\hline
\end{tabular}

Table II: Sensitivity, specificity and Youden indices of DCP, AFP, CA-199 and combination of DCP+AFP+CA-199.

\begin{tabular}{lccc}
\hline Tumor biomarker & Sensitivity $(\%)$ & Specificity $(\%)$ & Youden index \\
\hline DCP $(\mathrm{mAU} / \mathrm{ml})$ & 65.38 & 84.62 & 0.5 \\
AFP $(\mathrm{ng} / \mathrm{ml})$ & 50 & 92.31 & 0.423 \\
CA-199 $(\mathrm{U} / \mathrm{ml})$ & 26.92 & 90.38 & 0.173 \\
DCP+AFP+CA-199 & 92.31 & 75 & 0.673 \\
\hline DCP: Des-gamma-carboxyprothrombin, AFP: Alpha-fetoprotein, CA-199: & Carbohydrate
\end{tabular}

DCP: Des-gamma-carboxyprothrombin, AFP: Alpha-fetoprotein, CA-199: Carbohydrate antigen-199. 


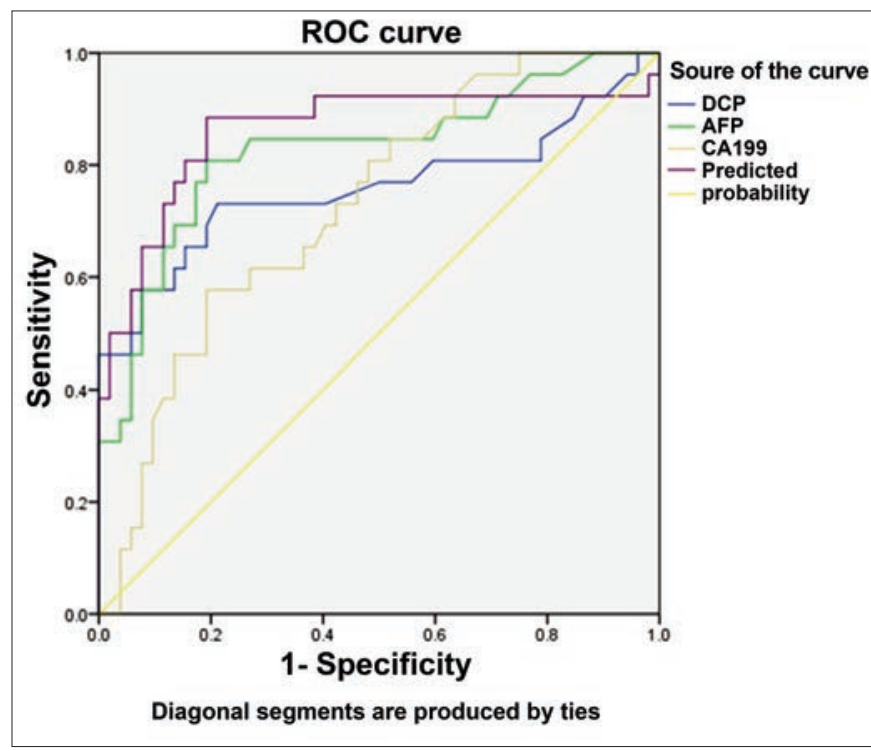

Figure 1: ROC curve of DCP, AFP, CA-199, and combination of DCP+AFP+ CA-199.

AUC: Area under the receiver operating characteristic curve.

$\mathrm{Cl}$ : Confidence intervals.

DCP: $A U C=0.759(95 \% \mathrm{Cl}: 0.624-0.894)$

AFP: $A U C=0.826(95 \% \mathrm{Cl}: 0.720-0.933)$

CA-199: $A U C=0.727(95 \%$ Cl: $0.613-0.841)$

Predicted probability (combination of DCP+AFP+CA-199): $A \cup C=0.862(95 \%$ Cl: 0.755-0.968).

respectively. The combination of DCP, AFP, and CA-199 exhibited the best diagnostic efficacy, with a Youden index of 0.673 (Table II).

DCP, AFP, and CA-199 showed diagnostic efficacy with AUC values of $0.759,0.826$ and 0.727 , respectively, while combination of DCP, AFP, and CA-199 demonstrated the best diagnostic efficacy, with AUC values of 0.862 (Figure 1). Moreover, compared with the single biomarker, combinations of biomarkers increased the diagnostic efficacy.

\section{DISCUSSION}

This study focused primarily on the efficacy of DCP, AFP and CA-199 measurements for diagnosis of CHCC-CC. The incidence, immunohistochemical biomarkers, and diagnostic efficacy of imaging and frozen section examination for $\mathrm{CHCC}-\mathrm{CC}$ were also briefly explored.

As a kind of very rare tumor with incidence of as low as $0.23 \%, 11$ there are few studies with limited cases on the research of $\mathrm{CHCC}-\mathrm{CC}$ in previous literatures, and even fewer studies on its diagnostic markers. AFP, DCP and CA-199 have been studied in a research of 10 cHCC-CC cases with 2 cases demonstrating simultaneous elevation of AFP/DCP and CA-19911; and the increase of both serum AFP and CA19-9 may be considered the suspicion of CHCC-CC 1 . Up to now, there seems no special research for the diagnostic efficacy of serum markers.

The Youden index and AUC are the most frequently used parameters for measurement of diagnostic efficacy, 12,13 with higher values of both parameters indicating superior efficacy. ${ }^{14}$ In this study, both methods were used to explore and compare the diagnostic efficacy of DCP, AFP and CA-199 in patients with CHCC-CC. The Youden indices for DCP and AFP indicated good diagnostic accuracy. AUC values range from 0.5 (indicating accuracy equivalent to that expected by chance) to 1 (denoting perfect accuracy). DCP, AFP, and CA-199 had diagnostic efficacy for $\mathrm{CHCC}-\mathrm{CC}$ with AUC values much greater than 0.5. The combination of DCP, AFP, and CA199 exhibited the best diagnostic efficacy, with the highest Youden index and AUC value. Overall, the Youden indices and $A U C$ values led to the conclusion that DCP, AFP, and CA-199 showed good diagnostic efficacy for $\mathrm{CHCC}-\mathrm{CC}$ and that combination of these three biomarkers could improve their diagnostic value.

Previous studies have reported conflicting clinical characteristics of $\mathrm{cHCC}-\mathrm{CC}$. Some studies found that cHCC-CC was more related to HCC than to $\mathrm{CC}$, based on factors including age, sex, serum AFP level, hepatitis virus infection, and liver cirrhosis, $5,15-17$ while other studies have reported that $\mathrm{CHCC}-\mathrm{CC}$ is closely associated with $\mathrm{CC}$, rather than $\mathrm{HCC}$, based on genetic, molecular biological characteristics and prognosis.2,3,11 The diagnostic markers for HCC (DCP and AFP) had higher efficacy than that associated with CC (CA-199) in our study, indicating that $\mathrm{CHCC}-\mathrm{CC}$ may be more closely related to $\mathrm{HCC}$ than to $\mathrm{CC}$. Compared with their diagnostic efficacy for HCC, as determined in a systematic review, both DCP and AFP had highly similar Youden index and AUC values for CHCC-CC. 18

The overall incidence of $\mathrm{CHCC}-\mathrm{CC}$ in a study of over 20,000 patients with PLC was 1.3\%.1 However, incidence has varied markedly among different areas from $0.23 \%$ to $14.2 \% .2,11,15$ Based on our study of 1,656 cases of PLC, $40(2.42 \%)$ cases of CHCC-CC were diagnosed by histological examination and 26 cases with full records were included for further analysis. Notably, some HCC or CC cases which do not undergo surgery maybe misdiagnosed by imaging alone; hence, the true incidence of $\mathrm{CHCC}-\mathrm{CC}$ in our sutdy should be above $2.42 \%$.

Although cHCC-CC was classified by Allen and Lisa as early as in 1949, little is known about its clinical characteristics. ${ }^{19}$ Clinicians are not particularly familiar with the imaging characteristics, ${ }^{8}$ frozen section examination or the method of biopsy (e.g., sampling only the area of $\mathrm{HCC}$ or $\mathrm{CC}$ within a $\mathrm{CHC}$ ) for $\mathrm{CHCC}-\mathrm{CC}$. This can directly affect the management strategy and prognosis. For cHCC-CC, lymph node dissection is necessary in surgery and liver transplantation may not be considered. Combined surgery with multimodal therapy is needed to improve the survival.

As a rare and aggressive kind of PLC with significant biological and histological heterogeneity, cHCC-CC 
remains a diagnostic challenge before surgery/biopsy. 20 In this study, we found that $\mathrm{CHCC}-\mathrm{CC}$ was more likely to happen in elderly male patients with cirrhosis. All patients were diagnosed by enhanced computed tomography (CT), magnetic resonance imaging (MRI) or both before surgery/biopsy; however, no case was diagnosed with cHCC-CC accurately by these imaging examinations. Twenty-five cases were diagnosed with PLC and one case was misdiagnosed with CC. In all 26 cases of cHCC-CC with full records, four males were not suitable for surgery in the preoperative assessment (the overall physical condition, anatomical characteristics, the extent of the tumor and so on) and were diagnosed with $\mathrm{cHCC}-\mathrm{CC}$ by biopsy (one refused further treatment because of old age and the other three cases underwent transarterial chemoembolization because of advanced stage). The remaining 22 cases underwent surgery directly without prior biopsy. The diagnostic efficacy of intraoperative frozen section examination was also very low. In this study, only $45.5 \%$ (10/22) cases were diagnosed correctly by frozen section examination, which means only 10 cases received the proper operative method (hepatectomy and lymph node dissection). The other 11 cases underwent partial hepatectomy without lymph node dissection and one case underwent liver transplantation. The low diagnostic efficacy of imaging or frozen section examinations represents a very serious phenomenon. As a consequence, 12 of 22 cases may not have received the appropriate type of surgery (hepatectomy without lymph node dissection or liver transplantation), since lymph node dissection is necessary and liver transplantation should not be considered for $\mathrm{CHCC}-\mathrm{CC} .4,6$

Surgical resection is considered the only curative option for cHCC-CC and aggressive surgical treatment including lymph node dissection could improve survival. 21 The reported variation in incidence and low diagnostic efficacy of imaging and frozen section examination emphasise the importance of the development of serum biomarkers.

Similarly, no standardisation of immunohistochemical biomarkers has been established. Glypican-3 (GPC-3) is a useful immunohistochemical biomarker for $\mathrm{HCC}, 22$ and cytokeratin 19 (CK19) is a valuable immunohistochemical biomarker for CC.23 In this study, GPC-3, CK19, hepatocyte AFP, cytokeratin 34 (CD34), and cytokeratin 7 (CK7) were examined. High ratios of positivity for CK19 (24/26) and GPC-3 (19/26) were founded in cHCC-CC by immunohistochemical staining. Other immunohistochemical biomarkers, such as hepatocyte AFP, CD34, and CK7, were either positive or negative. The immunohistochemical staining strategy for $\mathrm{cHCC}-\mathrm{CC}$ cases also needs to be standardised in the future. Although patients with $\mathrm{cHCC}-\mathrm{CC}$ were matched to controls based on age, gender, liver texture and ethnicity, selection bias may have existed in this study given the rarity of $\mathrm{cHCC}-\mathrm{CC}$. Future analysis of higher numbers of cases and prospective studies are required.

\section{CONCLUSION}

With high diagnostic efficacy, good reproducibility, ease of implementation, objectivity and non-invasiveness, DCP, AFP and CA-199 are ideal biomarkers and their combination measurement could be considered an easily accessible complement to imaging and frozen section examination for diagnosis of cHCC-CC, especially in elderly male patients with cirrhosis.

\section{ETHICAL APPROVAL:}

The study was approved prior to initiation of the research work by the Ethics Committees of the First Affiliated Hospital, College of Medicine, Zhejiang University.

\section{PATIENTS' CONSENT:}

As an observational correlational study, a waiver for informed consent was obtained from the Ethics Committees of the First Affiliated Hospital, College of Medicine, Zhejiang University and all the data from patients were analysed anonymously.

\section{CONFLICT OF INTEREST:}

Authors declared no conflict of interest.

\section{AUTHORS' CONTRIBUTION:}

GY: Contributed to the conception and design of this work.

YL: Contributed to data analysis and revised it critically for important intellectual content.

JC: Approved the final version to be published and agreed to be accountable for all aspects of the work ensuring that questions related to the accuracy or integrity of any part of the work are investigated and resolved appropriately.

\section{REFERENCES}

1. O'Connor K, Walsh JC, Schaeffer DF. Combined hepatocellular-cholangiocarcinoma (cHCC-CC): a distinct entity. Ann Hepatol 2014; 13:317-22.

2. Wang AQ, Zheng YC, Du J, Zhu CP, Huang HC, Wang SS, et al. Combined hepatocellular cholangiocarcinoma: Controversies to be addressed. World J Gastroenterol 2016; 22: 4459-65.

3. Maximin S, Ganeshan DM, Shanbhogue AK, Dighe MK, Yeh MM, Kolokythas $\mathrm{O}$, et al. Current update on combined hepatocellular-cholangiocarcinoma. Eur J Radiol Open 2014; 1:40-8.

4. Magistri P, Tarantino G, Serra V, Guidetti C, Ballarin R, Di Benedetto F. Liver transplantation and combined hepatocellular-cholangiocarcinoma: Feasibility and outcomes. Digestive and liver disease. Dig Liver Dis 2017; 49:467-70.

5. Lee $\mathrm{CH}$, Hsieh SY, Chang CJ, Lin YJ. Comparison of clinical characteristics of combined hepatocellular-cholangiocarcinoma and other primary liver cancers. J Gastroenterol Hepatol 2013; 28:122-7. 
6. Okuda K. Natural history of hepatocellular carcinoma including fibrolamellar and hepato-cholangiocarcinoma variants. J Gastroenterol Hepatol 2002; 17:401-5.

7. Schlageter M, Terracciano LM, D'Angelo S, Sorrentino P. Histopathology of hepatocellular carcinoma. World J Gastroenterol 2014; 20:15955-64.

8. Gera S, Ettel M, Acosta-Gonzalez G, Xu R. Clinical features, histology, and histogenesis of combined hepatocellularcholangiocarcinoma. World J Hepatol 2017; 9:300-9.

9. Li Y, Chen J. Serum des-gamma-carboxy prothrombin for diagnosis of adult primary cancer in liver. $J$ Coll Physicians Surg Pak 2019; 29:972-6.

10. Liu Y, Sun J, Zhang Q, Jin B, Zhu M, Zhang Z. Identification of bile survivin and carbohydrate antigen 199 in distinguishing cholangiocarcinoma from benign obstructive jaundice. Biomark Med 2017; 11:11-8.

11. Jung DH, Hwang S, Kim KH, Hong SM, Lee YJ, Ahn CS, et al. Clinicopathological features and post-resection prognosis of double primary hepatocellular carcinoma and intrahepatic cholangiocarcinoma. World J Surg 2017; 41:825-34.

12. Alemayehu D, Zou KH. Applications of ROC analysis in medical research: recent developments and future directions. Acad Radiol 2012; 19:1457-64.

13. Chen F, Xue Y, Tan MT, Chen P. Efficient statistical tests to compare youden index: accounting for contingency correlation. Statis Med 2015; 34:1560-76.

14. Xiong $X$, Yan $P$, Gao $C$, Sun $Q$, Xu F. The value of contrastenhanced ultrasound in the diagnosis of cesarean scar pregnancy. Biomed Res Int 2016; 2016:4762785.

15. Kassahun WT, Hauss J. Management of combined hepatocellular and cholangiocarcinoma. Int J Clin Prac 2008; 62:1271-8.
16. Fujii $H$, Zhu XG, Matsumoto $T$, Inagaki $M$, Tokusashi $Y$, Miyokawa N, et al. Genetic classification of combined hepatocellular-cholangiocarcinoma. Hum Pathol 2000; 31: 1011-7.

17. Li R, Yang D, Tang CL, Cai P, Ma KS, Ding SY, et al. Combined hepatocellular carcinoma and cholangiocarcinoma (biphenotypic) tumors: clinical characteristics, imaging features of contrastenhanced ultrasound and computed tomography. BMC Cancer 2016; 16:158.

18. Li C, Zhang Z, Zhang P, Liu J. Diagnostic accuracy of desgamma-carboxy prothrombin versus alpha-fetoprotein for hepatocellular carcinoma: A systematic review. Hepatol Res 2014; 44:E11-25.

19. Li Z, Wu X, Bi X, Zhang Y, Huang Z, Lu H, et al. Clinicopathological features and surgical outcomes of four rare subtypes of primary liver carcinoma. Clin J Cancer Res 2018; 30:364-72.

20. Stavraka C, Rush H, Ross P. Combined hepatocellular cholangiocarcinoma (cHCC-CC): an update of genetics, molecular biology, and therapeutic interventions. J Hepatocell Carcinoma 2018; 6:11-21.

21. Kim KH, Lee SG, Park EH, Hwang S, Ahn CS, Moon DB, et al. Surgical treatments and prognoses of patients with combined hepatocellular carcinoma and cholangiocarcinoma. Ann Surg Oncol 2009; 16:623-9.

22. Geramizadeh B, Seirfar N. Diagnostic value of arginase-1 and glypican-3 in differential diagnosis of hepatocellular carcinoma, cholangiocarcinoma and metastatic carcinoma of liver. Hepat Mon 2015; 15:e30336.

23. Jain R, Fischer S, Serra S, Chetty R. The use of cytokeratin 19 (ck19) immunohistochemistry in lesions of the pancreas, gastrointestinal tract, and liver. Appl Immunohistochem Mol Morphol 2010; 18:9-15. 